\title{
Solitary wave solutions of the fourth order Boussinesq equation through the $\exp (-\Phi(\eta))$-expansion method
}

\author{
M Ali Akbar ${ }^{1 *}$ and Norhashidah $\mathrm{Hj}$ Mohd $\mathrm{Ali}^{2}$
}

\begin{abstract}
The $\exp (-\Phi(\eta))$-expansion method is an ascending method for obtaining exact and solitary wave solutions for nonlinear evolution equations. In this article, we implement the $\exp (-\Phi(\eta))$-expansion method to build solitary wave solutions to the fourth order Boussinesq equation. The procedure is simple, direct and useful with the help of computer algebra. By using this method, we obtain solitary wave solutions in terms of the hyperbolic functions, the trigonometric functions and elementary functions. The results show that the $\exp (-\Phi(\eta))$-expansion method is straightforward and effective mathematical tool for the treatment of nonlinear evolution equations in mathematical physics and engineering.
\end{abstract}

Keywords: $\exp (-\Phi(\eta))$-expansion method; Fourth order Boussinesq equation; Solitary wave solutions; Soliton; Traveling wave solutions

Mathematics subject classifications: $35 \mathrm{C07} ; 35 \mathrm{C08} ; 35 \mathrm{P} 99$

\section{Background}

The world around us is inherently nonlinear (He 2009) and nonlinear evolution equations (NLEEs) are widely used as models to describe complex physical phenomena in various fields of science and engineering, especially in solid-state physics, plasma physics, fluid mechanics, biology etc. One of the fundamental problems for these models is to obtain their travelling wave solutions as well as solitary wave solutions. In particular, various methods have been utilized to explore different kinds of solutions of physical problems described by nonlinear evolution equations. In the numerical methods, stability and convergence should be considered, so as to avoid divergence or inappropriate results. However, in recent times, a variety of analytical and semi-analytical methods have been developed and use for solving NLEEs, for instance, the inverse scattering transform (Ablowitz and Clarkson 1991), the complex hyperbolic function method (Chow 1995; Zayed et al. 2006), the rank analysis method (Feng 2000), the ansatz method (Hu 2001a, b),

\footnotetext{
*Correspondence: ali_math74@yahoo.com

'Department of Applied Mathematics, University of Rajshahi, Rajshahi, Bangladesh

Full list of author information is available at the end of the article
}

the $\left(G^{\prime} / G\right)$-expansion method (Wang et al. 2008; Bekir 2008; Neyrame et al. 2012; Akbar et al. 2012; Alam and Akbar 2013; Alam et al. 2014), the Exp-functions method (He and Wu 2006), the modified simple equation method (Jawad et al. 2010; Khan et al. 2013), the Jacobi elliptic function method (Chen and Wang 2005; Liu 2005), the Adomian decomposition method (Adomian 1994; Wazwaz 2002), the homogeneous balance method (Wang 1995; Zayed et al. 2004), the F-expansion method (Wang and Zhou 2003; Wang and Li 2005), the Backlund transformation method (Miura 1978), the Darboux transformation method (Matveev and Salle 1991), the homotopy perturbation method (Mohyud-Din 2007; Mohyud-Din and Noor 2009), the generalized Riccati equation method (Yan and Zhang 2001), the tanh-function method (Wazwaz 2005), the Hirota's bilinear method (Hirota 2004), the auxiliary equation method (Sirendaoreji 2007), the $\exp (-\Phi(\eta))$-expansion method (Khan and Akbar 2013) etc.

The objective of this article is to implement the potential $\exp (-\Phi(\eta))$-expansion method to search solitary wave solutions for nonlinear evolution equations via the fourth order Boussinesq equation. In former literature, the 
solitary wave solutions to the Boussinesq equation have not been studied by this method.

The article is organized as follows: In Methodology Section, we give the description of the $\exp (-\Phi(\eta))$-expansion method. We apply this method to the fourth order Boussinesq equation in the Application Section. 2D and 3D graphs are given in the Graphical Representations of the Solutions Section. Finally, in Conclusion Section, we draw our conclusions.

\section{Methodology}

Let us consider the nonlinear evolution equation in the form

$$
F\left(u, u_{t}, u_{x}, u_{x x}, u_{t t}, u_{t x}, \ldots\right)
$$

where $u=u(x, t)$ is an unknown function, $F$ is a polynomial in $u(x, t)$ and its derivatives in which highest order derivatives and nonlinear terms are involved and the subscripts indicate partial derivatives. In order to investigate solitary wave solutions of (1) by using the exp $(-\Phi(\eta))$-expansion method, we have to perform the following important steps:

Step 1 . We combine the real variables $x$ and $t$ by a compound variable $\eta$

$$
u(x, t)=u(\eta), \quad \eta=x \pm V t
$$

where $V$ is the celerity of the traveling wave. By means of traveling wave transformation (2), Eq. (1) switch into an ordinary differential equation (ODE) for $u=u(\eta)$ :

$$
H\left(u, u^{\prime}, u^{\prime \prime}, u^{\prime \prime \prime}, \cdots\right)
$$

where $H$ is a polynomial of $u$ and its derivatives and the superscripts refer to the ordinary derivatives with respect to $\eta$.

Step 2. Assume the traveling wave solution of (3) can be articulated as follows:

$$
u(\eta)=\sum_{i=0}^{N} A_{i}(\exp (-\Phi(\eta)))^{i}
$$

$A_{i}(0 \leq i \leq N)$ are constants to be determined, such that $A_{N} \neq 0$ and $\Phi=\Phi(\eta)$ satisfies the following auxiliary equation:

$$
\Phi^{\prime}(\eta)=\exp (-\Phi(\eta))+\mu \exp (\Phi(\eta))+\lambda
$$

Depending on the parameters involved, Eq. (5) has the subsequent solutions:

When $\mu \neq 0$, and $\lambda^{2}-4 \mu>0$,

$$
\Phi(\eta)=\ln \left(\frac{-\sqrt{\left(\lambda^{2}-4 \mu\right)} \tanh \left(\frac{\sqrt{\left(\lambda^{2}-4 \mu\right)}}{2}(\eta+E)\right)-\lambda}{2 \mu}\right)
$$

When $\mu \neq 0$, and $\lambda^{2}-4 \mu<0$,

$$
\Phi(\eta)=\ln \left(\frac{\sqrt{\left(4 \mu-\lambda^{2}\right)} \tan \left(\frac{\sqrt{\left(4 \mu-\lambda^{2}\right)}}{2}(\eta+E)\right)-\lambda}{2 \mu}\right)
$$

When $\mu=0, \lambda \neq 0$, and $\lambda^{2}-4 \mu>0$,

$$
\Phi(\eta)=-\ln \left(\frac{\lambda}{\exp (\lambda(\eta+E))-1}\right)
$$

When $\mu \neq 0, \lambda \neq 0$, and $\lambda^{2}-4 \mu=0$,

$$
\Phi(\eta)=\ln \left(-\frac{2(\lambda(\eta+E)+2)}{\lambda^{2}(\eta+E)}\right)
$$

When $\mu=0, \lambda=0$, and $\lambda^{2}-4 \mu=0$,

$$
\Phi(\eta)=\ln (\eta+E)
$$

Step 3. The positive integer $N$ can be determined by considering the balance between the highest order
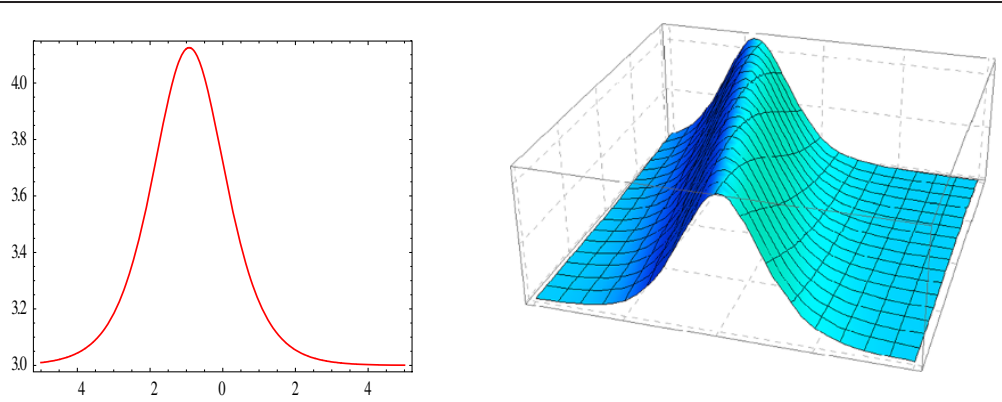

Figure 1 Bell shaped 2D and 3D-plot of the solitary wave. The graphs have been plotted from $u_{1}(\eta)$ when $\mu=1, \lambda=2.5, E=0, t=0$ and $A_{0}=1$. 

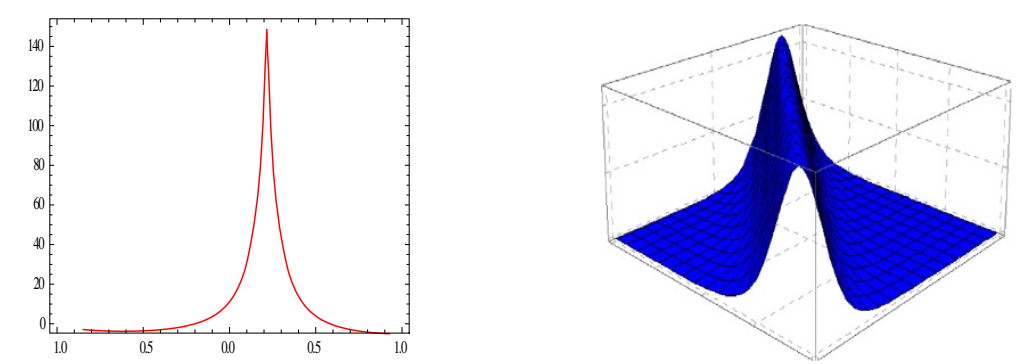

Figure 2 Sharp bell shaped 2D and 3D-plot of the solitary wave. The graphs have been plotted from $u_{2}(\eta)$ when $\mu=2.5, \lambda=1, E=0, t=0$ and $A_{0}=1$.

derivatives and the nonlinear terms of the highest order appearing in (3).

Step 4. We substitute Eq. (4) into Eq. (3) and then we take into consideration the function $\exp (-\Phi(\eta))$. In consequence of this substitution, we obtain a polynomial in $\exp (-\Phi(\eta))$. We collect all the coefficients of identical power of $\exp (-\Phi(\eta))$ and equalize to zero delivers a system of algebraic equations whichever can be solved to find $A_{N}, \cdots \cdots, V, \lambda, \mu$. The values of $A_{N}, \cdots \cdots, V, \lambda, \mu$ along with general solutions of Eq. (5) complete the determination of the solution of Eq. (1).

\section{Application}

In this section, we will use the $\exp (-\Phi(\eta))$-expansion method to construct the exact solutions and then the solitary wave solutions to the fourth order Boussinesq equation. Let us consider the equation

$$
u_{t t}-u_{x x}-u_{x x x x}-3\left(u^{2}\right)_{x x}=0 .
$$

The above model (11) was introduced by Boussinesq to illustrate the propagation of long waves in shallow water (Lai et al. 2008), where $u(x, t)$ is the elevation of the free surface of the fluid, where the subscripts denoting partial derivatives. The equation also arises in many other physical applications, such as, nonlinear lattice waves, iron sound waves in plasma, and vibrations in a nonlinear string. It was also applied to the study of the percolation of water in porous subsurface strata.

Equation (11) possesses solitary waves, extract from traveling wave solutions and Boussinesq was the first who gave a scientific explanation of their existence. We utilize the traveling wave variable $u(\eta)=u(x, t), \eta=x-V t$ and this operation changes (11) to the following ODE:

$$
V^{2} u^{\prime \prime}-u^{\prime \prime}-u^{(4)}-3\left(u^{2}\right)^{\prime \prime}=0
$$

Integrating Eq. (12) twice with respect to $\eta$ yields:

$$
\left(V^{2}-1\right) u-u^{\prime \prime}-3 u^{2}+C=0,
$$

where $C$ is an integration constant to be determined.

Balancing the highest order nonlinear term $u^{2}$ and linear term of the highest order $u$ " appearing in (13), yields $N=2$. Therefore, the solution of Eq. (13) takes the form

$$
u(\eta)=A_{0}+A_{1} \exp (-\Phi(\eta))+A_{2} \exp (-\Phi(\eta))^{2}
$$

where $A_{0}, A_{1}, A_{2}$ are arbitrary constants such that $A_{2} \neq 0$.

We substitute Eq. (14) into Eq. (13) and taking consideration Eq. (5), it generates a polynomial and then setting the coefficients of $\exp (-\Phi(\eta))$ to zero, yields

$$
\begin{aligned}
& -6 A_{2}-3 A_{2}^{2}=0 \\
& -2 A_{1}-10 A_{2} \lambda-6 A_{1} A_{2}=0 \\
& -A_{2}-4 A_{2} \lambda^{2}-3 A_{1} \lambda-3 A_{1}{ }^{2}-6 A_{0} A_{2}+V^{2} A_{2}-8 A_{2} \mu=0 \\
& C+V^{2} A_{0}-A_{0}-2 A_{2} \mu^{2}-3 A_{0}{ }^{2}-3 A_{1}{ }^{2}-A_{1} \lambda \mu=0
\end{aligned}
$$

Solutions of Eqs. (17)-(20), yield
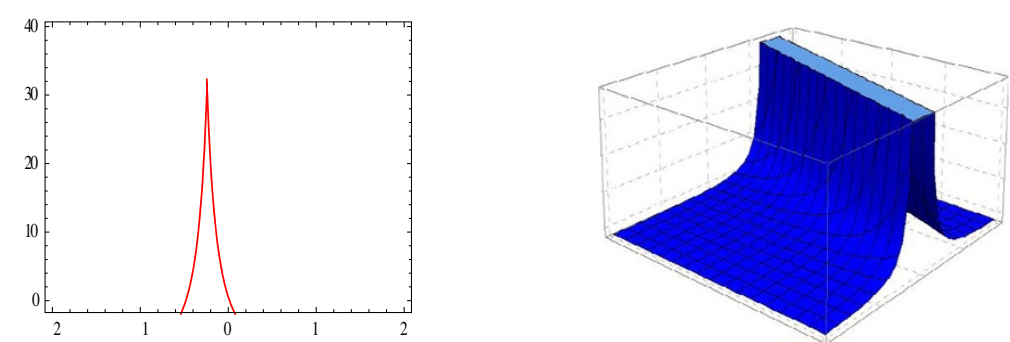

Figure 3 Pointed 2D and 3D-plot of the solitary wave. The graphs have been plotted from $u_{3}(\eta)$ when $\mu=0, \lambda=1, E=1, t=0$ and $A_{0}=1$. 

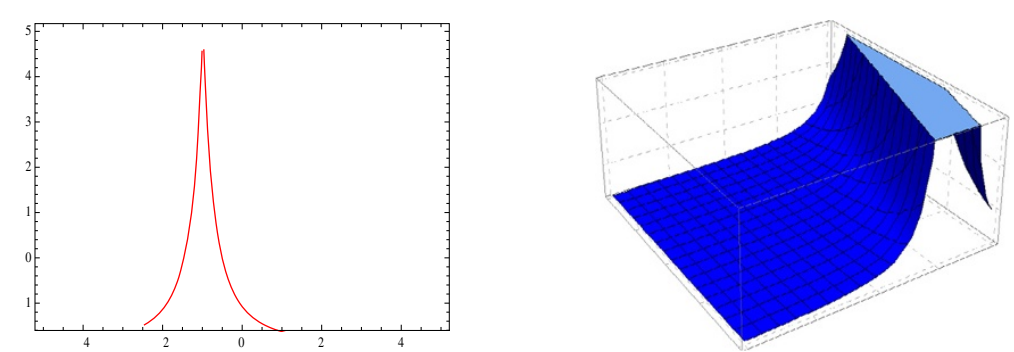

Figure 4 Sharp bell shaped 2D and 3D-plot of the solitary wave. The graphs have been plotted from $u_{4}(\eta)$ when $\mu=0.25, \lambda=1, E=1, t=0$ and $A_{0}=1$.

$$
\begin{aligned}
& C=-4 \mu^{2}-3 A_{0}^{2}-2 \lambda^{2} \mu-\lambda^{2} A_{0}-8 A_{0} \mu \\
& V= \pm \sqrt{\lambda^{2}+1+8 \mu+6 A_{0}}, A_{1}=-2 \lambda, A_{1}=-2
\end{aligned}
$$

where $\lambda, \mu$ and $A_{0}$ are arbitrary constants.

Substituting the values of $V, A_{0}, A_{1}, A_{2}$ into Eq. (14), yields

$$
u(\eta)=A_{0}-2 \lambda \exp (-\Phi(\eta))-2 \exp (-\Phi(\eta))^{2}
$$

where $\eta=x \mp \sqrt{\lambda^{2}+1+8 \mu+6 A_{0}}$.

By using the solutions of Eq. (5) into Eq. (21), we obtain the succeeding traveling wave solutions of the fourth order Boussinesq equation:

Type 1: When $\mu \neq 0, \lambda^{2}-4 \mu>0$,

$$
\begin{aligned}
u_{1}(\eta)= & A_{0}+\frac{4 \lambda \mu}{\sqrt{\lambda^{2}-4 \mu} \tanh \left(\frac{\sqrt{\lambda^{2}-4 \mu}}{2}(\eta+E)\right)+\lambda} \\
& -\frac{8 \mu^{2}}{\left\{\sqrt{\lambda^{2}-4 \mu} \tanh \left(\frac{\sqrt{\lambda^{2}-4 \mu}}{2}(\eta+E)\right)+\lambda\right\}^{2}},
\end{aligned}
$$

where $\eta=x \mp \sqrt{\lambda^{2}+8 \mu+6 A_{0}+1} t$ and $E$ is an arbitrary constant.

Type 2 : When $\mu \neq 0, \lambda^{2}-4 \mu<0$,

$$
\begin{aligned}
u_{2}(\eta) & =A_{0}-\frac{4 \lambda \mu}{\sqrt{4 \mu-\lambda^{2}} \tan \left(\frac{\sqrt{4 \mu-\lambda^{2}}}{2}(\eta+E)\right)-\lambda} \\
& -\frac{8 \mu^{2}}{\left\{\sqrt{4 \mu-\lambda^{2}} \tan \left(\frac{\sqrt{4 \mu-\lambda^{2}}}{2}(\eta+E)\right)-\lambda\right\}^{2}},
\end{aligned}
$$

where $\eta=x \mp \sqrt{\lambda^{2}+8 \mu+6 A_{0}+1} t$ and $E$ is an arbitrary constant.

Type 3: When $\mu=0, \lambda \neq 0$, and $\lambda^{2}-4 \mu>0$,

$$
u_{3}(\eta)=A_{0}-\frac{2 \lambda^{2}}{\exp (\lambda(\eta+E))-1}-\frac{2 \lambda^{2}}{\{\exp (\lambda(\eta+E))-1\}^{2}},
$$

where $\eta=x \mp \sqrt{\lambda^{2}+8 \mu+6 A_{0}+1} t$ and $E$ is an arbitrary constant.

Type 4: When $\mu \neq 0, \lambda \neq 0$, and $\lambda^{2}-4 \mu=0$,

$$
u_{4}(\eta)=A_{0}+\frac{\lambda^{3}(\eta+E)}{\lambda(\eta+E)+2}-\frac{1}{2}\left(\frac{\lambda^{2}(\eta+E)}{\lambda(\eta+E)+2}\right)^{2},
$$

where $\eta=x \mp \sqrt{\lambda^{2}+8 \mu+6 A_{0}+1} t$ and $E$ is an arbitrary constant.

Type 5: When $\mu=0, \lambda=0$, and $\lambda^{2}-4 \mu=0$,

$$
u_{5}(\eta)=A_{0}-\frac{2}{(\eta+E)^{2}}
$$

where $\eta=x \mp \sqrt{\lambda^{2}+8 \mu+6 A_{0}+1} t$ and $E$ is an arbitrary constant.
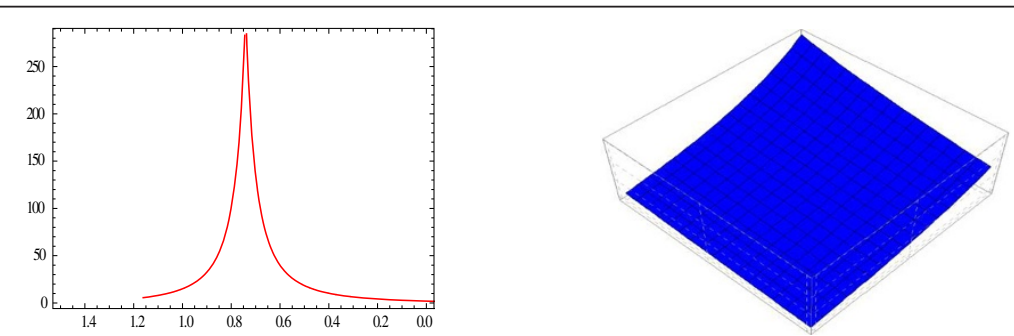

Figure 5 2D and 3D-plot of the solitary wave. The graphs have been plotted from $u_{5}(\eta)$ when $\mu=0, \lambda=0, E=1, t=0$ and $A_{0}=1$. 
Solitary wave solutions represent an important type of solutions for nonlinear partial differential equations (PDEs) as many nonlinear partial differential equations have been found to have a variety of solitary wave solutions. It is familiar that searching of exact solutions of nonlinear partial differential equations plays a significant role in the study of nonlinear physical phenomena. Exact traveling wave solutions are useful for verifying the accuracy and stability of popular numerical schemes such as the finite difference and finite element methods. The solitary wave solutions obtained in this article are encouraging, applicable, and could be helpful in analyzing long wave propagation on the surface of a fluid layer under the action of gravity, iron sound waves in plasma, and vibrations in a nonlinear string.

\section{Graphical representation of the solutions}

Solitary waves can be obtained from each traveling wave solution by setting particular values to its unknown parameters. By adjusting these parameters, one can get an internal localized mode. In this section, we have presented some graphs of solitary waves constructed by taking suitable values of the involved unknown parameters to visualize the underlying mechanism to the original physical phenomena. Using mathematical software Mathematica, two and three-dimensional plots of the obtained solutions have been shown in Figures 1, 2, 3,4 and 5 .

The obtained solutions of the fourth order Boussinesq equation incorporate four types of explicit solutions namely hyperbolic, trigonometric, exponential, and rational function solutions. From these explicit results we observe that solutions $u_{1}(\eta)$ and $u_{2}(\eta)$ are soliton and the rest of the three solutions are cuspon. The above solitary wave solutions might be useful in analyzing the propagation of long waves in shallow water, iron sound waves in plasma, and vibrations in a nonlinear string.

\section{Conclusions}

In this article, we have successfully formulated solitary waves solutions from the traveling wave solutions to the fourth order Boussinesq equation through the exp $(-\Phi(\eta))$-expansion method. The procedure is simple, direct and constructive with the help of a computer algebra system. The method is quite efficient and practically well suited to be used in finding solitary wave solutions of NLEEs and the attained solutions demonstrated the competence of the $\exp (-\Phi(\eta))$-expansion method. We also observed that the method is straightforward and can be applied to many other nonlinear evolution equations.

\section{Competing interests}

The authors declare that they have no competing interests.

\section{Authors' contributions}

The authors, viz MAA and NHMA with the consultation to each other carried out this work and drafted the manuscript together. Both authors read and approved the final manuscript.

\section{Acknowledgement}

The authors wish to take this opportunity to express their sincere gratitude to the anonymous referees for their valuable comments and suggestions.

\section{Author details}

${ }^{1}$ Department of Applied Mathematics, University of Rajshahi, Rajshahi, Bangladesh. ${ }^{2}$ School of Mathematical Sciences, Universiti Sains Malaysia, Pulau Pinang, Malaysia.

Received: 3 May 2014 Accepted: 10 June 2014

Published: 8 July 2014

\section{References}

Ablowitz MJ, Clarkson PA (1991) Soliton, nonlinear evolution equations and inverse scattering. Cambridge University Press, New York

Adomian G (1994) Solving frontier problems of physics: the decomposition method. Kluwer Academic Publishers, Boston

Akbar MA, Ali NHM, Zayed EME (2012) A generalized and improved (G'/G)expansion method for nonlinear evolution equations. Math Probl Eng 2012, doi:10.1155/2012/459879

Alam MN, Akbar MA (2013) Exact traveling wave solutions of the KP-BBM equation by using the new generalized $\left(G^{\prime} / G\right)$-expansion method. Springerplus 2:617, doi:10.1186/2193-1801-2-617

Alam MN, Akbar MA, Mohyud-Din ST (2014) A novel ( $\left.G^{\prime} / G\right)$-expansion method and its application to the Boussinesq equation. Chin Phys B 23(2):020203

Bekir A (2008) Application of the ( $\left.G^{\prime} / G\right)$-expansion method for nonlinear evolution equations. Phys Lett A 372:3400-3406

Chen Y, Wang Q (2005) Extended Jacobi elliptic function rational expansion method and abundant families of Jacobi elliptic functions solutions to (1+1)dimensional dispersive long wave equation. Chaos Solitons Fract 24:745-757

Chow KW (1995) A class of exact periodic solutions of nonlinear envelope equation. J Math Phys 36:4125-4137

Feng X (2000) Exploratory approach to explicit solution of nonlinear evolutions equations. Int J Theor Phys 39:207-222

He JH (2009) Nonlinear science as a fluctuating research frontier. Chaos Solitons Fract 41:2533-2537

He JH, Wu XH (2006) Exp-function method for nonlinear wave equations. Chaos Solitons Fract 30:700-708

Hirota R (2004) The direct method in soliton theory. Cambridge University Press, London

$\mathrm{Hu} J \mathrm{~L}$ (2001a) Explicit solutions to three nonlinear physical models. Phys Lett A 287:81-89

$\mathrm{Hu} J \mathrm{~L}$ (2001b) A new method for finding exact traveling wave solutions to nonlinear partial differential equations. Phys Lett A 286:175-179

Jawad AJM, Petkovic MD, Biswas A (2010) Modified simple equation method for nonlinear evolution equations. Appl Math Comput 217:869-877

Khan K, Akbar MA (2013) Application of $\exp (-(\varphi(\xi)))$-expansion method to find the exact solutions of modified Benjamin-Bona-Mahony equation. World Appl Sci J 24(10):1373-1377

Khan K, Akbar MA, Alam MN (2013) Traveling wave solutions of the nonlinear Drinfel'd-Sokolov-Wilson equation and modified Benjamin-Bona-Mahony equations. J Egyptian Math Soc 21:233-240

Lai S, Wu YH, Zhou Y (2008) Some physical structures for the (2+1)-dimensional Boussinesq water equation with positive and negative exponents. Comput Math Appl 56:339-345

Liu D (2005) Jacobi elliptic function solutions for two variant Boussinesq equations. Chaos Solitons Fract 24:1373-1385

Matveev VB, Salle MA (1991) Darboux transformation and solitons. Springer, Berlin

Miura MR (1978) Backlund transformation. Springer, Berlin

Mohyud-Din ST (2007) Homotopy perturbation method for solving fourth-order boundary value problems. Math Probl Eng 2007, doi:10.1155/2007/98602

Mohyud-Din ST, Noor MA (2009) Homotopy perturbation method for solving partial differential equations. Zeitschrift fur Naturforschung A-A J Phys Sci 64a:157-170 
Neyrame A, Roozi A, Hosseini SS, Shafiof SM (2012) Exact travelling wave solutions for some nonlinear partial differential equations. J King Saud Uni Sci 22:275-278

Sirendaoreji (2007) Auxiliary equation method and new solutions of Klein-Gordon equations. Chaos Solitions Fract 31:943-950

Wang M (1995) Solitary wave solutions for variant Boussinesq equations. Phys Lett A 199:169-172

Wang ML, Li XZ (2005) Extended F-expansion method and periodic wave solutions for the generalized Zakharov equations. Phys Lett A 343:48-54

Wang ML, Zhou YB (2003) The periodic wave solutions for the Klein-GordonSchrodinger equations. Phys Lett A 318:84-92

Wang ML, Li XZ, Zhang J (2008) The (G'/G)-expansion method and traveling wave solutions of nonlinear evolution equations in mathematical physics. Phys Lett A 372:417-423

Wazwaz AM (2002) Partial differential equations: method and applications. Taylor and Francis, London

Wazwaz AM (2005) The tanh method for generalized forms of nonlinear heat conduction and Burgers-Fisher equations. Appl Math Comput 169:321-338

Yan Z, Zhang H (2001) New explicit solitary wave solutions and periodic wave solutions for Whitham Broer-Kaup equation in shallow water. Phys Lett A 285(5-6):355-362

Zayed EME, Zedan HA, Gepreel KA (2004) On the solitary wave solutions for nonlinear Hirota-Sasuma coupled KDV equations. Chaos Solitons Fract 22:285-303

Zayed EME, Abourabia AM, Gepreel KA, Horbaty MM (2006) On the rational solitary wave solutions for the nonlinear Hirota-Satsuma coupled KdV system. Appl Anal 85:751-768

doi:10.1186/2193-1801-3-344

Cite this article as: Akbar and Ali: Solitary wave solutions of the fourth order Boussinesq equation through the $\exp (-\Phi(\eta))$-expansion method. SpringerPlus 2014 3:344.

\section{Submit your manuscript to a SpringerOpen ${ }^{\circ}$ journal and benefit from:}

- Convenient online submission

- Rigorous peer review

- Immediate publication on acceptance

- Open access: articles freely available online

- High visibility within the field

- Retaining the copyright to your article

Submit your next manuscript at $>$ springeropen.com 